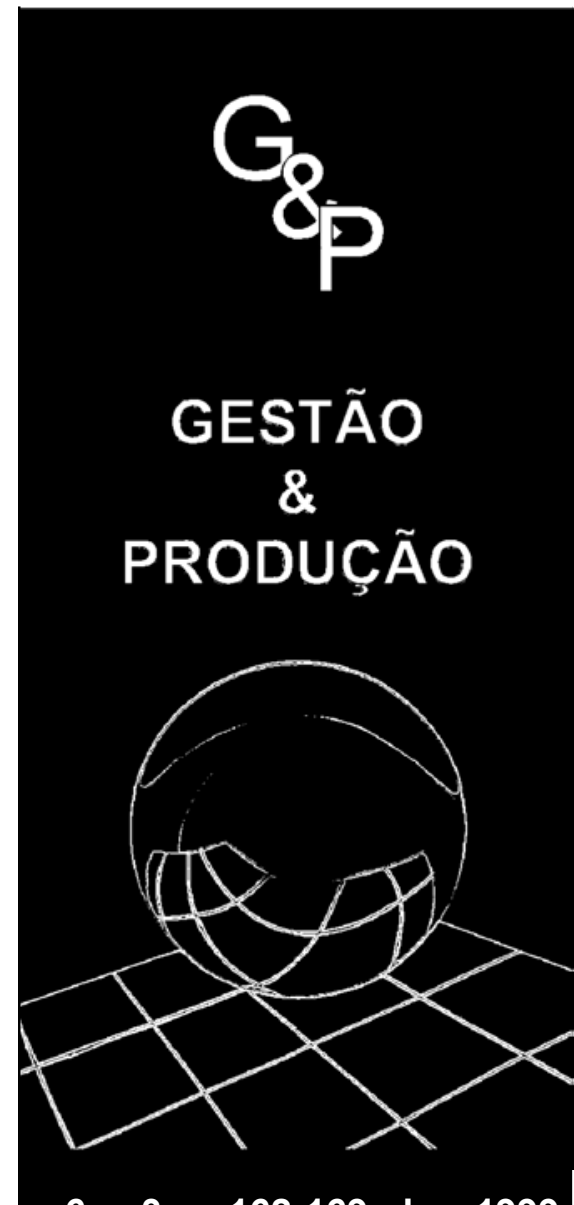

\title{
INVESTIGAÇÃO EMPÍRICA DA EFICIÊNCIA NO SETOR DE ALIMENTOS
}

v.6, n.3, p. 162-169, dez. 1999

Paulo Sergio Ceretta

Departamento de Ciências Administrativas Universidade Federal de Santa Maria E-mail: rs019458@pro.via-rs.com.br

\section{Resumo}

Intensivos processos de integração de mercados, conjuntamente com a profunda transformação que tem ocorrido com o sistema econômico mundial, justificam a avaliação da eficiência do setor de alimentos no Brasil. O propósito deste estudo é de analisar a Eficiência Técnica Total, Eficiência Técnica e a Eficiência de Escala. Para tal, são utilizados os modelos de análise por envoltório de dados. Muitas empresas foram classificadas como ineficientes e esta ineficiência indica o potencial para a gestão da empresa desenvolver melhorias na performance em relação aos seus referenciais.

\section{Palavras-chave: eficiência, desempenho, análise por envoltório de dados.}

\section{Introdução}

$\mathrm{N}$ ão há dúvidas que do processo de formação de grandes blocos econômicos ou com a própria globalização dos negócios, surgem duas conseqüências diretas para as empresas: (1) aumento do mercado consumidor - potencial de consumo - e com ele a possibilidade do lançamento de novos produtos; (2) aumento do nível da concorrência no que se refere aos atuais consumidores, que passarão a ter uma maior variedades/opções de produtos e serviços similares ao seu alcance, muitos deles com tecnologia superior e melhores preços.

Para fazer frente a estas duas questões atuais, os gestores de empresas procuram, cada vez mais, não apenas se manterem atualizados mas desenvolverem processos, técnicas e produ- 
tos/serviços criativos e inovadores. De certa forma, isto torna possível ser mais administrável o risco do negócio frente ao ambiente turbulento e competitivo. Esta realidade não é diferente no Setor de Alimentos, onde, a cada dia que passa, as empresas enfrentam uma maior oferta de opções de produtos, tanto nacionais como importados, exigindo delas fortes investimentos no desenvolvimento de novos produtos e principalmente na capacidade gerencial.

Neste cenário, parece haver a dúvida se o lucro seria uma medida adequada de avaliação do desempenho empresarial no curto prazo/ médio prazo, como propõe BIERMAN (1994). A moderna gestão empresarial apresenta-se cada vez mais inclinada a atuar sobre conceitos de desempenho ou expectativas de desempenho e não sobre o enfoque de resultados fiscais e valores puramente monetários.

Entende-se desempenho organizacional como uma combinação de diversos resultados desejados gerados a partir de um processo. A palavra que parece estar complicando o entendimento deste conceito (simples e elementar) é como combinar resultados desejados de grandezas totalmente diferentes, por exemplo: como combinar satisfação dos clientes/público com o lucro, a participação de mercado com resultados financeiros. Observe que estes últimos são de mesma grandeza, porém, de natureza e enfoque estratégico totalmente distintos.

Uma conseqüência direta deste novo enfoque é a possibilidade de se ampliar grandemente as potencialidades do planejamento e desenvolvimento organizacional. Muito mais do que lucro, o empresário está preocupado em diagnosticar se existem fontes de ineficiência dentro da organização, a fim de amenizar sua ação sobre o desempenho pretendido. É evidente que melhorar é preciso, porém, "não podemos melhorar nossa situação competitiva se não houver um diagnóstico preciso da situação atual, como estamos e qual a fonte de nossa possível ineficiência; também, é necessário obter informações semelhantes sobre o desempenho das outras empresas do setor, após, ajusta-se a política da empresa conforme a futura ação e influência de variáveis ambientais controláveis e não controláveis".

Este trabalho tem por objetivo analisar o desempenho das empresas do Setor de Alimentos, identificando as organizações eficientes e as ineficientes e, para estas últimas, identificando, também, o grau e a fonte de ineficiência. Isto fornece subsídios sólidos para a gestão da empresa iniciar o desenvolvimento de um processo de melhoria. Os diversos trabalhos existentes na literatura sobre quantificação da eficiência têm sido classificados em dois grandes grupos:

- Trabalhos que se utilizam de técnicas de regressão para estimar uma fronteira de produção estocástica e/ou paramétrica (são os modelos econométricos); e

- Pesquisas que se apóiam em modelos de programação matemática não paramétrica para identificar uma superfície eficiente de produção.

Obviamente, cada um destes enfoques alternativos apresenta suas vantagens e desvantagens. Uma excelente discussão sobre esta questão pode ser obtida em FORSUND, LOVELL \& SCHMIDT (1980). O desenvolvimento desta pesquisa fica limitado à utilização de modelos não paramétricos de avaliação da Eficiência Técnica Total, Eficiência Técnica e Eficiência de Escala.

\section{Medida Não Paramétrica de Eficiência}

耳 studos sobre eficiência, utilizando-se de Cuma abordagem não paramétrica, têm seu início com o notável trabalho de FARRELL (1957). FARRELL foi o pioneiro na decomposição da eficiência produtiva em dois outros componentes: eficiência alocativa e eficiência técnica. Para desenvolver seu estudo, assumiu duas importantes hipóteses: é possível diminuir determinada quantidade de insumo sem alterar o nível de produção e/ou aumentar o consumo de outro insumo (descarte forte), a tecnologia subjacente ao estudo era tida como de retorno constante de escala. 
Posteriormente, CHARNES, COOPER \& RHODES (1978), influenciados pelas idéias de FARRELL, introduziram a metodologia de Análise por Envoltório de Dados, do Inglês Data Envelopment Analysis - DEA. A Análise por Envoltório de Dados é uma metodologia de programação matemática não paramétrica, desenvolvida com o objetivo de avaliar a eficiência técnica de um conjunto de unidades, que, tradicionalmente, são referenciadas na literatura como Decision Making Units - DMUs.

Na Análise por Envoltório de Dados sobre um conjunto de $n$ DMUs é determinada uma fronteira eficiente representativa dos melhores desempenhos e possíveis combinações destes; esta também é interpretada como uma superfície eficiente de produção. Nesta avaliação, as unidades que estão sobre a superfície são ditas como eficientes, por outro lado, as unidades que estão abaixo desta são chamadas de ineficientes, sendo possível identificar uma medida de eficiência técnica para cada unidade analisada. Basicamente, a medida de eficiência obtida quantifica a distância entre a unidade analisada e o seu ponto projetado sobre a superfície eficiente.

Inicialmente a grande maioria das aplicações de DEA estavam relacionadas com a avaliação da eficiência de órgãos públicos, hospitais e universidades. Mais recentemente, parece haver uma tendência de sua utilização na avaliação de empresas privadas (BEASLEY, 1998) e principalmente instituições bancárias (NOULAS \& MILLER, 1990; SHERMAN \& LADINO, 1995; PARADI et al., 1997; THANASSOULIS, 1999). Porém, existem inúmeras outras aplicações da metodologia DEA, nesse sentido uma importante fonte de informação é a bibliografia disponibilizada por SEIFORD (1990).

O objetivo central da Análise por Envoltório de Dados é de identificar comparativamente o grau de eficiência de uma determinada unidade, sendo esta ineficiente; identificar, também, a fonte desta ineficiência. Desta maneira, os gestores da unidade terão subsídios para desenvolver processos de melhoria de desempenho sustentados em bases sólidas, possibilitando-lhes alterar o atual plano de operação.

É necessário, para introduzir os modelos DEA, esclarecer alguns aspectos da notação a serem utilizados - optou-se por utilizar a notação mais comum na literatura especializada. São consideradas e analisadas $n$ DMUs; cada uma destas consome quantidades variadas de $m$ diferentes insumos objetivando gerar quantidades variadas de $s$ diferentes tipos de resultados desejados. Então, uma unidade específica $k$ consome quantidade $\mathrm{x}_{\mathrm{ik}}>0$ de insumo $i$ e produz quantidade $\mathrm{y}_{\mathrm{rk}}>0$ de resultado desejado $r$. Desta forma, $X_{k}$ e $Y_{k}$ são os vetores de insumo e resultado da unidade $k$, enquanto que $\mathbf{X}$ representa a matriz $n \times m$ de insumos e $\mathbf{Y}$ a matriz $n \times s$ de resultados para o conjunto de $n$ unidades.

O modelo DEA desenvolvido por CHARNES, COOPER \& RHODES - CCR (1978), é dado pela seguinte formulação:

$$
\begin{array}{cl}
\operatorname{Max} & \theta+\varepsilon(\mathbf{1 s}+\mathbf{1 e}) \\
\text { s.t. } & \mathbf{Y}_{\lambda}-\mathrm{s}=\theta \mathrm{Y}_{\mathrm{k}} \\
& \mathbf{X}_{\lambda}+\mathrm{e}=\mathrm{X}_{\mathrm{k}} \\
& \lambda \geq 0, \mathrm{~s} \geq 0, \mathrm{e} \geq 0
\end{array}
$$

Posteriormente, BANKER, CHARNES \& COOPER - BCC (1984), pela da adição da restrição de convexidade ao modelo anteriormente descrito, ou seja $(\mathbf{1} \lambda=1)$, desenvolveram uma formulação para identificar a eficiência técnica. $O$ valor de $\theta$, em ambas as formulações, identifica o nível de eficiência das unidades em relação ao seu conjunto de referência, que repousa sobre a superfície eficiente. Porém, o valor de $\theta$ no modelo CCR é utilizado para identificar a Eficiência Técnica Total, enquanto que o valor de $\theta$ no modelo BCC identifica apenas a Eficiência Técnica. Esses termos serão discutidos na próxima seção.

\section{Decomposição da Eficiência Técnica Total}

$\mathrm{O}$ sentido da palavra "eficiência", utilizada neste trabalho, é em relação ao quanto melhor uma unidade utiliza um vetor de insumos 


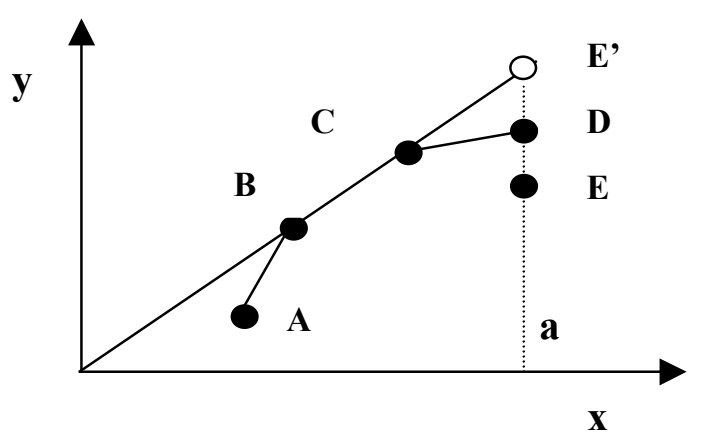

Figura 1 - Relação Insumo-Resultado e Superfície Eficiente.

(processo) para gerar um vetor de resultados. Como foi dito na seção anterior, FARRELL (1957) foi o pioneiro nesta área, demonstrando que a eficiência técnica total pode ser decomposta em duas outras medidas: eficiência técnica e eficiência alocativa. Suas idéias foram posteriormente aperfeiçoadas nos modelos CCR e BCC.

Uma ilustração do relacionamento entre a utilização de um único insumo x para a obtenção de um único resultado y é dado pela Figura 1. Os pontos $\mathrm{A}, \mathrm{B}, \mathrm{C}, \mathrm{D}$ e $\mathrm{E}$ representam unidades organizacionais e suas diversas combinações empíricas de $\mathrm{x}$, y. A superfície eficiente do modelo CCR é aquela determinada pela linha que une os pontos $0 \mathrm{E}^{\prime}$. Já para o modelo BCC, a superfície eficiente é a linha que une os pontos ABCD.

A Eficiência Técnica Total, para a empresa representada pelo ponto $\mathrm{E}$, é dada pela razão da distância de $\mathrm{aE} / \mathrm{aE}$. Este cálculo considera que as proporções entre as variáveis observadas permanecerão constantes para qualquer porte de organização. $O$ índice de eficiência representa o grau de alavancagem que projetará a unidade observada até a superfície eficiente.

Por outro lado, o indicador de Eficiência Técnica é obtido pela razão da distância de $\mathrm{aD} / \mathrm{aE}$. Neste caso, a hipótese de retorno constante é relaxada, ou seja, considera-se que unidades de porte diferentes possam operar com produtividades distintas. $\mathrm{O}$ índice de eficiência identifica o valor, cujos resultados, da empresa analisada, deverão ser multiplicados, projetando- a até a superfície eficiente. A Eficiência Técnica é também, entendida como uma habilidade puramente gerencial por considerar restrições mais flexíveis quanto à manutenção de produtividade em diferentes níveis de operação.

E, por fim, a Eficiência de Escala é obtida pela razão da distância de aE'/aD. O índice de Eficiência de Escala identifica o ganho de produtividade que a organização pode obter caso for possível alterar a sua escala de operação, passando a operar em uma região de maior produtividade. Estes vários indicadores de eficiência podem ser combinados na seguinte equação:

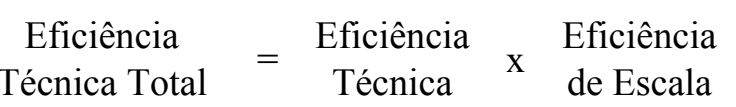

Agora considere o caso em que um único resultado y é gerado a partir da utilização de dois insumos $\mathrm{x}_{1}$ e $\mathrm{x}_{2}$. Esta representação está expressa na Figura 2. As unidades analisadas estão representadas pelos pontos F, G, H e I. A superfície eficiente está representada pela linha que une os pontos FGH. É fácil verificar que a empresa I é ineficiente por estar abaixo desta superfície, o seu indicador de eficiência é dado por 0I'/0I. Porém, observe que o ponto $\mathrm{F}$ garante o mesmo nível de operação em relação ao insumo $\mathrm{x}_{1}$ mas utiliza menor quantidade do insumo $\mathrm{x}_{2}$. Logo, a unidade I terá que, além de alavancar o nível de produção até o seu ponto referência I' sobre a superfície, reduzir o nível de consumo do insumo $x_{21}$, na proporção de $\mathrm{x}_{2 \mathrm{~F}}-\mathrm{x}_{2 \mathrm{I}}$, garantindo, assim, a posição de eficiência dada pelo ponto $\mathrm{F}$. 


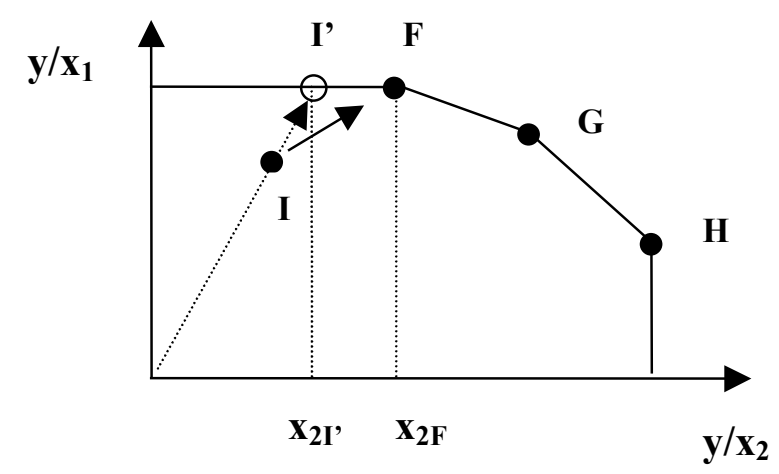

Figura 2 - Superfície Eficiente e o Problema de Descarte.

A situação evidenciada na Figura 2, de reduzir o nível de determinado insumo sem alterar o nível de produção projetado e sem aumentar a utilização do outro insumo, pode ser entendida como um problema de "descarte" de insumo e está relacionada com o desbalanceamento na relação resultado/insumo, procurando um melhor aproveitamento dos fatores. Em outras palavras, o valor do descarte está associado com ineficiência adicional específica de algum(s) insumo(s). Para maiores detalhes, ver BYRNES, FÄRE \& GROSSKOPF (1984); outro excelente texto é FÄRE, GROSSKOPF \& LOVELL (1985).

\section{Metodologia}

$\mathrm{O}$ dados utilizados para o desenvolvimento deste trabalho foram obtidos junto à revista "Exame - Melhores e Maiores", e fazem referência a 21 empresas classificadas pela publicação como sendo do Setor de Alimentos. As empresas que constituem a amostra são as seguintes: Sadia/SC, Leite Paulista/SP, Granol/SP, Açúcar União/SP, Central/MG, Quaker/SP, Aurora/SC, Batavo/PR, Braswey/SP, Danone/SP, Avipal/RS, Elegê/RS, Frangosul/RS, Sadia/PR, Cacique/PR, Itasa/MG, Plus/SP, Santa Elisa/SP, Aymoré/MG, Sudcoop/PR, Mococa/SP. Embora a amostra tenha sido estruturada de maneira não aleatória, acredita-se que seja representativa por se tratar de 21 grandes empresas do setor.

Como o objetivo do trabalho não está atrelado à identificação específica de cada empresa, optou-se por não identificá-las diretamente, utilizando-se para isso de códigos $\mathrm{EMP}_{1} \ldots$ $E_{21}$ A idéia inicial da pesquisa era de trabalhar uma aplicação mais forte da metodologia, utilizando múltiplos resultados desejáveis gerados a partir de múltiplos insumos; porém, como não havia muitas informações disponíveis, principalmente a respeito de aspectos qualitativos, utilizou-se apenas informações quantitativas publicadas.

As informações utilizadas como produto (resultados) referem-se ao conceito de Valor Adicionado (VA). Quanto aos insumos, foram considerados o Patrimônio Líquido (PL), Exigível (Exg.) e o montante de Salários (Sal.). Procurou-se avaliar as empresas pelas suas capacidades de gerar riqueza para os mais diversos públicos, os acionistas, os credores e funcionários da organização. Para um melhor entendimento do conceito de Valor Adicionado e geração de riqueza, ver NEVES \& VICECONTI (1998).

Obviamente, o aspecto que mais impõem limitações a este trabalho é a natureza dos dados utilizados. Na realidade, este é um fato crucial em todas as pesquisas empíricas realizadas no âmbito do analista externo, pois, no Brasil, as empresas limitam-se a tornarem público somente informações financeiras exigidas por lei. Desse modo, muitas variáveis importantes de cunho não financeiro não são levadas em consideração na análise. Isso, por sua vez, pode distorcer a interpretação dos resultados. 
Os indicadores de Eficiência Técnica Total (ETT) para cada empresa se referem ao valor de $\theta$ obtido pela aplicação da formulação CCR. E, para a Eficiência Técnica (ET), o valor de $\theta$ obtido pela aplicação da formulação BCC. Em ambos os casos, a empresa é considerada eficiente se o valor de $\theta$ for igual a unidade $(\theta=1)$, e ineficiente se for maior do que um $(\theta>1)$. A Eficiência de Escala (EE) é obtida pela simples razão entre a Eficiência Técnica Total e Eficiência Técnica $(\mathrm{EE}=\mathrm{ETT} / \mathrm{ET})$. Quanto aos valores do descarte, tanto na ETT como na ET, são obtidos pelos indicadores (s) e (e) em cada uma das respectivas formulações.

\section{Resultados Obtidos}

$\mathrm{O}$ resultados obtidos, em relação aos indicadores de Eficiência Técnica Total, Eficiência Técnica e Eficiência de Escala, bem como seus valores absolutos, estão resumidos na Tabela 1. Quanto à Eficiência Técnica Total, observe que, quatro (4) empresas apresentaram graus de alavancagem do Valor Adicionado igual à unidade $(C C R=1)$. Isto significa que elas estão sobre a superfície eficiente e, também, estão sendo utilizadas como referencial de comparação para as demais. Estas empresas, além de apresentarem uma capacidade gerencial superior às demais, estão operando em regiões de alta produtividade, não apresentando nenhum problema de ineficiência localizada (pelo menos em relação ao período analisado e às variáveis consideradas para análise). Neste sentido, podese dizer que estas organizações possuem uma facilidade muito grande de adaptarem-se às mudanças estruturais e comportamentais.

Em relação ao indicador de Eficiência Técnica, a situação é mais favorável, já que nove (9) empresas apresentaram grau de alavancagem igual à unidade $(\mathrm{BCC}=1)$. Por outro lado, identificou-se que, doze (12) das 21 empresas analisadas precisam repensar as suas atuais práticas; destas, $50 \%$ apresentam situação precária $(\mathrm{BCC}>1,40)$ somente para acompanhar o seu conjunto de referência. Também é possível verificar que, uma das maiores fontes de ineficiência do setor está relacionada com uma excessiva utilização de recursos de terceiros (Exg.), que, por conseqüência, eleva as despesas financeiras e o nível de risco empresarial, não havendo indícios de ineficiência quanto à despesas de pessoal.

E, por fim, no que se refere à questão de Eficiência de Escala, é notável que existem grandes dificuldades de manter idêntico desempenho para diferentes níveis de operação, pois apenas quatro (4) empresas não apresentaram este tipo de problemas.

\section{Conclusão}

$\mathrm{O}$ estudo da Eficiência Técnica Total, procedendo-se a sua decomposição em elementos de Eficiência Técnica, puramente gerencial, e elementos estruturais, permite à gestão de cada empresa um excelente diagnóstico do desempenho. Além de identificar o desempenho obtido, permite que se obtenha informações sobre o conjunto de referência, utilizado para comparação, e, também, as fontes de ineficiência (se houver). No caso das organizações apresentarem Ineficiência Técnica, estes indicadores passam a ser fortes subsídios para o desenvolvimento de programas de melhoria técnica. Já as empresas que apresentaram apenas Ineficiência de Escala, as informações levam a uma discussão mais profunda sobre aspectos de investimentos de longo prazo, políticas e redirecionamento do negócio.

Especificamente no setor de alimentos, no período analisado, conclui-se que existe uma enorme área para atuação gerencial sobre a questão de ineficiência na geração de riqueza. Neste aspecto, deve-se salientar, contrariamente ao que geralmente é divulgado, a ineficiência não está diretamente relacionada às questões de despesa de pessoal, o que, de certa forma, vem ao desencontro de programas de demissões de pessoal como alternativa de melhorar o desempenho, estando, portanto, mais relacionado com o excesso de aplicação de recursos onerosos. 
Tabela 1 - Indicadores de Eficiência Obtidos para as 21 Empresas do Setor de Alimentos. Período de 1997.

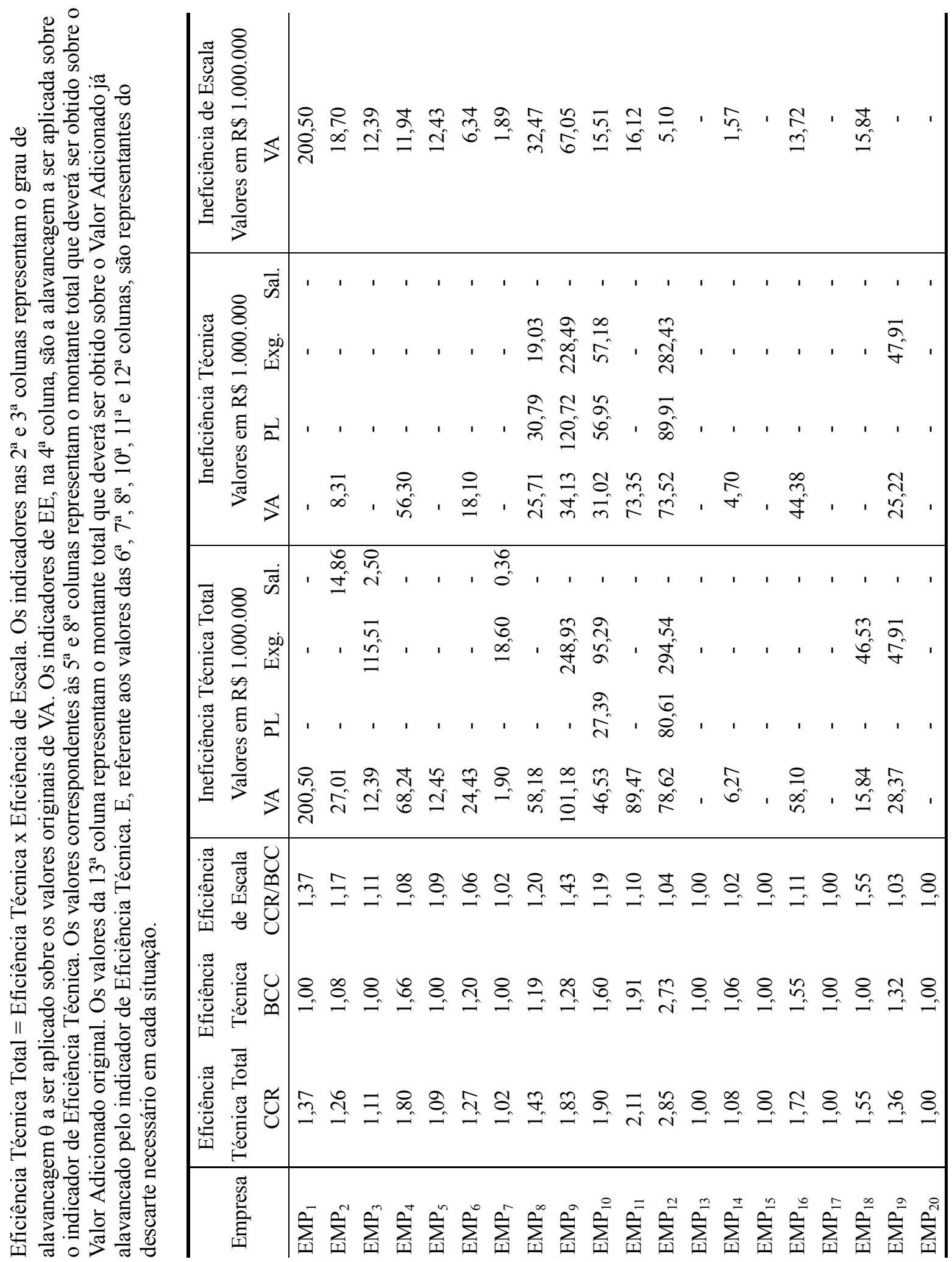




\section{Referências Bibliográficas}

BANKER, R.D.; CHARNES, A. \& COOPER, W.W.: "Some models for estimating technical and scale inefficiencies in data envelopment analysis." Management Science, 30, p.1078-1092, 1984.

BEASLEY, J.E.: “Allocating fixed cost and resources via data envelopment analysis." Working paper, The Management School - Imperial College, London/England, 1998.

BIERMAN Jr., H.: "Accounting for valuation and evaluation." The Journal of Portfolio Management. Spring, p.64-67, 1994.

BYRNES, P.; FÄRE, R. \& GROSSKOPF, S.: "Measuring productive efficiency: an application to illinois strip mines." Management Science, 30, p.671-681, 1984.

CHARNES, A.; COOPER, W.W. \& RHODES, E.: "Measuring the efficiency of decision making units." European Journal of Operational Research, 2, p.429-444, 1978.

FÄRE, R.; GROSSKOPF, S. \& LOVELL, C.A.K.: Measurement of efficiency of production. Boston: Kluwer-Nijhoff, 1985.

FARREL, M.J.: "The measurement of productive efficiency." Journal of the Royal Statistical Society. Series A, 120, p.253-281, 1957.
FORSUND, F.; LOVELL, C.A.K. \& SCHMIDT, P.: "A survey of frontier productions and their relationship of efficiency measurement." Journal of Econometrics, 13, p.5-26, 1980.

NEVES, S. \& VICECONTI, P.E.V.: Contabilidade avançada. São Paulo: Frase Editora. 1998.

NOULAS, A.G. \& MILLER, S.: "The technical efficiency of large bank production." Journal of Banking \& Finance, 20, p.495-509, 1996.

PARADI, J.C.; ROSEN, D. \& SCHAFFNIT, C.: "Best practice analysis of bank branches: an application of DEA in a large Canadian bank." European Journal of Operational Research, 98, p.269-286, 1997.

REVISTA EXAME. Melhores e maiores. São Paulo: Editora Abril. Julho - 1998.

SEIFORD, L.M.: “A bibliography of data envelopment analysis (1978-1990)." Working paper, University of Massachusetts at Amherst, 1990.

SHERMAN, H.D. \& LADINO, G.: "Managing bank productivity using data envelopment analysis (DEA)." Interfaces, 25, p.60-73, 1995.

THANASSOULIS, E.: "Data envelopment analysis and its uses in banking." Interfaces, 29, p.1-13, 1999.

\section{AN EMPIRICAL STUDY OF THE EFFICIENCY IN THE FOOD SECTOR}

\section{Abstract}

The intensive process of markets integration, together with the profound transformation that has taken place in the economic system worldwide, justifies the evaluation of efficiency in the food sector in Brazil. The aim of this study is to analyze Overall Technical Efficiency, Technical Efficiency and Scale Efficiency. Data Envelopment Analysis models are used in this investigation. Many enterprises are classified as technically inefficient and slacks indicate the potential for management to improve the firm's performance relative to its benchmark.

Key words: efficiency, performance, data envelopment analysis. 